\title{
Lumen
}

Selected Proceedings from the Canadian Society for Eighteenth-Century Studies

\section{Newton's notion of matter in the 'De aere et aethere'}

\section{Laura Benítez}

Volume 25, 2006

URI : https://id.erudit.org/iderudit/1012074ar

DOI : https://doi.org/10.7202/1012074ar

Aller au sommaire du numéro

Éditeur(s)

Canadian Society for Eighteenth-Century Studies / Société canadienne d'étude du dix-huitième siècle

ISSN

1209-3696 (imprimé)

1927-8284 (numérique)

Découvrir la revue

Citer cet article

Benítez, L. (2006). Newton's notion of matter in the 'De aere et aethere'. Lumen, 25, 17-28. https://doi.org/10.7202/1012074ar

Copyright @ Canadian Society for Eighteenth-Century Studies / Sociéte canadienne d'étude du dix-huitième siècle, 2006
Ce document est protégé par la loi sur le droit d'auteur. L'utilisation des services d'Érudit (y compris la reproduction) est assujettie à sa politique d'utilisation que vous pouvez consulter en ligne.

https://apropos.erudit.org/fr/usagers/politique-dutilisation/ 


\section{Newton's notion of matter in the 'De ære et æthere'}

As a mechanical philosopher Newton knew that, no theory of matter could be firmly established until the forces effecting phenomena were thoroughly understood. ${ }^{1}$

\section{Introduction}

It is almost a commonplace that Newton did not want to face the problem of the constitution of matter. For some scholars, it is just a metaphysical and slippery problem far from the real concern of Newtonian physics. Nevertheless, in light of his own writings, this question arises as a very important one that occupied him and reveals his ontological preferences. So, it is important to make an accurate revision of his conception about matter and try to explain why, if he had in mind several options, he could not propose any of them as definitive.

In my opinion it is owing to the fact that Newton considered that none of them could fulfil his methodical requirements and not that he overlooked the importance of those hypotheses for physical science.

The brief essay 'De ære et æthere' is a collection of proposals about matter. Newton wrote it around 1673 and it constitutes, according to the Halls: 'The first surviving document fully devoted to this theory [of matter].'

1 Rupert Hall and Marie Boas Hall, Introduction to 'De ære et æthere,' Unpublished Scientific Papers of Isaac Newton, by Isaac Newton (Cambridge: Cambridge University Press, 1978), 211.

2 Hall, 'Introduction,' Scientific, 187. 
The essay includes the theory of ether and although it is difficult to agree with I.B. Cohen who says that it constitutes 'a central pillar of ... [Newton's] system of nature' neither is it something tentative, ${ }^{3}$ just like a bad thought that sometimes happened to him.

My own view is that Newton could not put forth a single theory of matter because he was trying diverse ways of explaining phenomena and the theory of ether was very important for mechanics in the epistemological way of reflection.

For Newton a very important problem was to find the direct connection between cohesion and density and their direct causes. But finally he substituted the explanation by means of causes by some very important mathematical constructions and in this respect the words of the Opticks still sound in our ears:

To tell us that every species of things is endowed with an occult specific quality by which it acts and produces manifest effects, is to tell us nothing; but to derive two or three general principles of motion from phenomena and afterwards to tell us how the properties and actions of all corporeal things follow from those manifest principles, would be a very great step in philosophy, though the causes of those principles were not yet discovered. ${ }^{4}$

My proposal here is that the diverse hypotheses of matter in Newton's 'De ære et aethere' had a very specific ontological importance and were substituted by theoretical constructions as the principles of motion, mathematically expressed. And that the 'great step in philosophy' to which Newton refers, can be viewed as his own change from the epistemological way of reflection, paradigmatically represented by Descartes, to the critical way of reflection proper of Newton's philosophy of nature, and also the change from mechanics to dynamics.

\section{From the epistemological to the critical way of reflection}

By 'way of reflection' I understand a 'mode' or 'form' of thought that different schools and authors sustain simultaneously or successively,

3 'For since Newton always presented his aetherial hypotheses tentatively, even in the height of his scientific prestige, there is no reason to suppose that he regarded them as other than tentative' (Hall, 'Introduction,' Scientific, 187).

4 Isaac Newton, Opticks, Quaery 31 (New York: Dover Publications, 1952) 401-402. 
even in different historical stages, grounded on some common assumptions. For this reason we can understand a 'way of reflection' as a kind of 'theoretical model,' although very basic and general, subjacent to diverse philosophical proposals.

The notion of 'way of reflection' is very flexible and it helps to avoid some problems, for example, to consider philosophy as a discontinuous subject. I consider that philosophical culture is a very complex web of 'ways of reflection' that we construct, enlarge, or stretch, and it depends on our problems and concerns.

In some sense, the 'way of reflection' is close to the notion of tradition, nonetheless it has to be restricted to very ample traditions.

In order to understand Newton's hypotheses on matter, I will contrast two ways of reflection that I will call the epistemological and the critical one.

The epistemological way of reflection is grounded on the assumption that the world is substantially homogeneous and that the only substance different from matter is consciousness that has the privilege to behold and to understand this material world, that is, consciousness can get to know it. The problem is, how can we understand the access of one substance, let us say the mind, to another, let us say matter, if they are really different? Or how, if the mind has only an immediate access to its ideas, can it possess a true real knowledge of the external world?

The 'critical way of reflection' assumes that we cannot have a hard ontology available to our knowledge. Therefore, the constitution of knowledge has to be grounded on the phenomenal experience; nevertheless the operations of the understanding (inference, imagination, etc.) must guide us to establish the regularities and principles that rule the diversity of phenomena that we experience.

So, the 'critical' in this way of reflection refers not only to a methodological rule, but also to a programme which allows us to construct (constitute) the objects of knowledge.

The critical programme tries, at the same time, to determine the limits of reason and the extent of the knowable reality. That is the reason why, in this way of reflection, it is impossible to accept the objects of knowledge as autonomous or independent data, because they have to be constituted or constructed by the mind.

Hence, the objects of knowledge as diverse 'theoretical corpus' are the most convincing explanations for a predetermined region of phenomenal effects. And it is for this last reason that the critical way of reflection is far away from the ideal of a one and only system of knowledge, and gives rise to diverse subject-matters as different areas or theoretical fields - articulated knowledge that exhibits its legality and is methodically grounded. 


\section{2. 'De aere et aethere' and Newtonian afflictions in the epistemological way of reflection}

In their commentary on Newton's 'De ære' the Halls assert that:

Newton's adherence to mechanical philosophy was a very early development. In his student days he has read with care the Principia Philosophiae of Descartes; The Origins of Forms and Qualities among many works of Boyle and the writings of Henry More and Robert Hooke, with others of the generation somewhat senior to his own. ${ }^{5}$

I want to underline not the obvious inheritance that Newton received from Descartes (the mechanism) or from Boyle (the atomism), but the fact that Newton has transited in his youth the epistemological way of reflection and the consequences it had for his natural philosophy.

'De ære et æthere' cannot be understood but as the product of Newton's transit by the epistemological way of reflection. In that way he acquired the idea of homogeneous matter and moreover, he took two important ideas of 'hard' ontology about matter:

1) That matter is divided in many imperceptible pieces whose existence can be inferred from the behaviour of the phenomena we have at hand.

2) The parts of air can be divided into even smaller parts, very subtle or ether.

As to the problem of material homogeneity, if each body (mineral, liquid, vegetal, animal, etc.) can be divided into smaller parts until these arrive to air, and air in turn can be divided in even smaller parts until it turns to ether, then Newton claims that there is a material homogeneity. But this principle is not only a Cartesian one; we can find it in the epistemological way of reflection since the lower Middle Ages and, more emphatically, in the Renaissance.

Newton alleges in the Conclusio that:

For the matter of all things is one and the same which is transmuted into countless forms by the operations of nature. ${ }^{6}$

5 'Introduction,' Scientific, 187.

6 Newton, Conclusio in op. cit., 341. 
The material homogeneity is very important if one desires to sustain that although the material particles are alike, their condensation, rarefaction, fermentation, and other physical as well as chemical processes explain the variety of bodies that we know.

On the other hand, this idea was opposed to the substance pluralism of the tradition and was characteristic of the epistemological way of reflection. But it is important to realise that the homogeneity as Newton proposes it, even if it in general follows 'the argument familiar to seventeenth-century mechanical philosophy: that all material bodies, including airs derived from them, are composed of particles' is not alike to Descartes's proposal grounded on his basic mechanism that reduces all physical explanation to collision among particles and cannot give an appropriate account of physiological phenomena as growth, nor of chemical reactions by proximity of bodies, or by heat as Newton does it.

Descartes's physico-geometrical proposal, that reduces air to spherical parts of 'medium' size and velocity becomes much more complex in Newton's view. Therefore in his 'De ære et æthere,' he says:

The threefold kind of air arises from the threefold kind of substances. Permanent and heavy air from metals, exhalation especially from the earthy part of vegetable substances and vapour from liquids. ${ }^{7}$

But for Newton it is not enough to mention the immediate origin of these kinds of air. He explains also how they function:

Moreover, aerial substances are very different according to the nature of the bodies from which they are generated. Metals by corrosion give true permanent air; vegetable and animal substances by corrosion, fermentation or burning give an air of short duration like an exhalation; and volatile substances rarefied by heat give an air least lasting of all, which we call a vapour. ${ }^{8}$

So, we are very far from the Cartesian explanation by means of the mere movement and collision of particles. In Newton's view there is an interplay of physical and chemical processes; hence, the notion of substance is, owing to this, closer to our idea of chemical substance than that of Aristotle. In fact, this new substantial plurality is not based on completely different natures, but on the consideration that the diverse op-

7 Newton, 'De ære,' 226.

8 Ibid. 
erations of nature give rise to the modification of matter into the variety of phenomena that we perceive.

Let us get now to the problem of 'basic ontology.' Related to the problem of the material parts, Newton states in Qu. 31 of his Opticks that:

[...] it seems probable to me, that God in the Beginning form'd Matter in solid, massy, hard impenetrable, moveable Particles $[\ldots]^{9}$

This is a categorical proposal since it states the existence of particles (or corpuscles) which are not even perceptible but one has to assume their existence in order to explain and understand phenomena. So at this point, he is closer to Descartes's corpuscularianism than to Berkeley's coloured extension, which is made out of minima sensibilia.

This closeness to Descartes's proposal at this point is made much more evident when Newton gets into the speculative explanation of ether. In fact, following quite closely the terminology of the Cartesian Principles of Philosophy, Newton says:

And just as bodies of this Earth by breaking into small particles are converted into air, so these particles can be broken into lesser ones by some violent action and converted into yet more subtle air which, if it is subtle enough to penetrate the pores of glass, crystal and other terrestrial bodies, we may call the spirit of air or the aether. ${ }^{10}$

Newton treats thus the subject of particle division approaching the Cartesian corpuscularian point of view. Nevertheless while for Descartes the divisibility of matter is inherent to his physico-geometrical conception, we ask ourselves why Newton, who privileges mechanics over geometry, is interested at a certain moment in this kind of proposal. I take it that here, Newton explores the explicative possibility of basic mechanics which he learnt as a young man and that he considers whether he can rescue an ontological 'hard core' conception to ground his own theory about attractive and repulsive forces. Even though the Cartesian explanation in terms of completely inert material particles, that gain and lose movement by collision, seemed to him insufficient to give an account of the great variety of natural phenomena. This allows us to

9 Newton, I., Opticks, Qu. 31 (New York: Dover Publications, 1952), 400.

10 Newton, I., De ære et æthere, 227. 
understand Newton's transit, from the epistemological to the critical way of reflection.

\section{Newton in the Critical Way of Reflection}

When Newton adds new elements as forces to material particles, he starts the transit towards the critical way of reflection. In this way he finally will find that it is better to leave aside the problems of hard ontology about matter, having to do with metaphysical principles, and substitute them by verifiable explanations that can be expressed mathematically.

In the 'De ære et æthere' Newton uncovers, besides two important ontological disagreements with Descartes, an even more important methodological one.

The ontological disagreements are:

1) That the material particles are not absolutely inert, i.e. they are not just vehicles or bearers of movement but that they have associated forces of attraction and repulsion.

2) That there is not a material plenum as Descartes proposed it, because from Newton's viewpoint condensation and rarefaction require empty space among moving material parts. Owing to this Newton's view comes closer to atomism.

The first disagreement, that is, that material parts are not merely inert, but that they have associated forces shows the transit from kinematics to dynamics. ${ }^{11}$

It is true that for Descartes any force of which its cause is not made explicit, is an occult one, but Newton finds that forces are rather explicit in phenomena as he states it in Query 31:

$[\ldots]$ it seems to me further that these particles have not only a vis inertiae, accompanied with such passive laws of motion as naturally result from that force, but also that they are moved by certain active principles, such as is that of gravity, and that which causes fermentation and the cohesion of bodies. ${ }^{12}$

11 The Halls say that: 'From the phenomena of physics, chemistry and even physiology, Newton concluded that matter can act upon matter in ways other than the direct mechanical impact of Cartesian physics' ('Introduction,' Scientific, 193).

12 Newton, Opticks, Qu. 31, 401. 
By not being able to obtain these phenomena from the mere mechanical collisions of the parts, Newton proposes the notion of active forces and also takes inertix as a passive force. Descartes had talked of particles in movement, but Newton introduces the notion of forces among parts. Now, what are forces and where do they take their origin?

These two questions receive in the 'De aere' an approximate treatment and not a complete and definitive explanation.

Newton takes forces to be active principles, powers or virtues, but does not go further in his ontological precision. Nonetheless he proposes two important characteristics: a) that forces do not inhere in matter, since God could have created matter without forces and so they are only associated to it and $b$ ) that there are non-mechanical forces, i.e. immaterial but physical, which can be studied experimentally as the gravitational force and that they are neither miracles nor spiritual forces. For the Halls, to admit immaterial but physical forces is an impossible metaphysics. ${ }^{13}$

I take it that forces have for Newton the very same intermediate ontological status as space since the latter is not material, it is simple and it exists with more ontological necessity than material objects, but it does not identify itself with the necessary being. With forces something similar happens: they are not material, but they are present and active among material particles, so they are not really occult forces but they are detectable and they occupy an intermediate place between the necessary being and the material and contingent beings.

As for the origin of forces, Newton adopts a peculiar academic scepticism with respect to the repulsive force. He proposes three reasonable hypotheses on the origin of this force but does not adopt any one of them, maintaining an academic scepticism pretty close to Hume's who does engage himself with the origin of ideas. Newton's tells us about the cause of repulsion:

Many opinions may be offered concerning the cause of this repulsion. The intervening medium may give way with difficulty or not suffer itself to be much compressed. Or God may have created a certain incorporeal nature which seeks to repel bodies and make them less packed together. Or it may be in the nature of bodies not only to have a hard and impenetrable nucleus, but also [to have] a certain surrounding sphere of most fluid and tenuous matter which admits other bodies into it with difficulty. ${ }^{14}$

13 Newton, 'De ære,' Scientific, 197-98.

14 Ibid, 223. 
But possibly the most important thing is that Newton considers it to be useless to argue about such speculations since what is really important are the effects:

About these matters I do not dispute at all. But as it is equally true that air avoids bodies, and bodies repel each other mutually, I seem to gather rightly from these that air is composed of the particles of bodies torn away from contact, and repelling each other with a certain large force. ${ }^{15}$

Related to the second disagreement, with respect to Cartesian mechanism, i.e., that there is no material plenum, it is grounded on the idea that there is no reason to identify matter with extension as Descartes does it. This problem is discussed by Newton at large in his 'De gravitatione et æquipondio fluidorum,' nevertheless, in his 'De ære et ætere' he expresses, with respect to air's rarefaction,

That under a half or a third or even a hundreth or a thousandth part of that [normal] weight [the air] is expanded to double or treble or even a hundred or a thousand times its normal space, which would hardly seem to be possible if the particles of air were in mutual contact. ${ }^{16}$

Hence, the basic assumption of the Newtonian thesis is that there is a void in which the material particles can move, i.e. to fly away from each other by means of the repulsion force.

Up to now we have only talked of what the Halls call the solid part of Newtonian physics:

That phenomena result from the motions of material particles, and that these motions are the result of the interplay of forces between the particles. ${ }^{17}$

The most interesting part is that this theory does not present itself from a hard ontological perspective, that is we do not know with much clarity whether Newton is talking of atoms or of corpuscles and nor where they come from and how they operate the forces associated to these particles. Newton seems to lose interest in those kind of explanations because he finds a more useful alternative.

15 Newton, 'De ære,' Scientific, 223.

16 Ibid.

17 Ibid, 192. 


\section{The Critical Way of Reflection Facing Metaphysics}

When we say that Newton adds forces to the particles or separates matter from extension, it seems that what is happening is that he found a better way of understanding movement or the change from Cartesian mechanics to dynamics. Nonetheless, the problem becomes more interesting under the light of his methodological proposal.

What makes plausible the change of the reflective way which Newton undertakes is precisely how he perceives the method of natural philosophy, since in founding its mathematical principles, he can become forgetful of the metaphysical ones - principles which were indispensable to Descartes up to the point that he said he had not read Galileo since the latter had not grounded his science on the general principles of the metaphysics.

Newton perceives something quite different. If the principles which ground the regularity of phenomena can have a proper mathematical expression, then one does not require metaphysical principles. This does not mean that Newton put completely aside the metaphysical support, especially when he goes by the epistemological reflective way: rather, the new science had no use for them.

The rejection of Cartesian physics is not really a matter of mere punctual rejections: it is stronger than that in the light of change of direction in the methodology. On this we read in the Scholium:

We see but the shapes and colours of bodies, we hear but sounds, we touch but external surfaces, we smell odours and taste flavours; but we know the substances or essences themselves by no sense, by no reflex action and therefore we have no more idea of them than a blind man has of colours. ${ }^{18}$

Descartes would have accepted the first parts, that is, 'but we know the substances or essences themselves by no sense'; nevertheless it is by a reflexive act that we come to know that things are not as they appear and certainly, innate ideas help us to know that extension is the essential mode of matter.

Newton establishes a gradation in the speculation. Of course, metaphysical speculation occupies the least place and it has to be excluded from the theory. But neither is admissible speculation in physics on mechanical causes, even though we know its effects, since Newton

18 Newton, I., Scholium Generale, Def. VIII, part IV, in op. cit., 361. 
admits in his Opticks that his philosophy is experimental, meaning that particular propositions are obtained from phenomena and afterwards they are generalized. In this way, if experiments are to confirm some principles or regularities in the behaviour of natural phenomena, such principles cannot be mere descriptions or forced analogies or, even worse, invented entities. The principles are restricted to certain proportional relations among specific aspects of phenomena, and their formulation has to be made in a rigorous manner, that is, mathematically. Hence the Halls state that:

But 'the mechanical principles' of the Principia require that the forces acting on particles and the motions produced by them be exactly calculated. ${ }^{19}$

Then, even though we do not know the hard ontology of particles and forces, what can be certainly established are the parameters of their behaviour. This is something which Newton expresses in the 'De ære et æthere':

But if by some principle acting at a distance [the particles] tend to recede mutually from each other, reason persuades us that the distance between their centres is doubled the force of recession will be halved, when trebled the force is reduced to a third and so on, and thus by an easy computation it is discovered that the expansion of the air is reciprocal to the compressive force. ${ }^{20}$

It remains clear that, for Newton, the method of natural philosophy substitutes the principles of metaphysics for mathematical principles and in so doing, an important consequence is obtained which is the rejection of the scheme of a unitary knowledge which was assumed to contain all the areas of knowledge grounded on a certain number of general principles. Now, instead one tries to establish an object of study as a sphere of knowledge perfectly defined by its object and by its method. This is how Newton goes from the epistemic way of reflection to the critical way of reflection and in this manner, he goes against the Cartesian scheme and attains the rejection of Leibniz.

But in this change of reflective way, the most important is not just to move away from Cartesianism but his attempt to put physics away from the metaphysical speculations. 


\section{Conclusions}

The Halls state that when Newton says, '[...] I have not yet disclosed the cause of gravity, nor I have undertaken to explain it, since I could not understand it from the phenomena, ${ }^{21}$ such a statement is partially true since Newton asserted that every force comes from God, that is, Newton could appeal to the metaphysical resource to establish the cause of gravity. The commentators speak of an ambiguity that is present throughout Newton's work.

I intend to qualify the fact: in my opinion, this is not a mere ambiguity; rather this is Newton's transit by two reflective ways, one that accepts metaphysical principles and another one which questions them. But besides this, the success of the new science is marked by the substitution of the metaphysical principles by the mathematical ones or, if one so desires, by the substitution of speculative hypotheses by theoretical terms as is the case in not going too deep into the nature of gravity and postulate it backing it by the regular behaviour of phenomena and within a mathematical scheme.

Briefly, the separation of space from material parts, the transformation of mechanism into dynamics, and the constitution of natural philosophy as a subject of study which separates from a hard metaphysical basis, constitute in Newton the transit from the epistemological way of reflection to the critical way of reflection and all this can be seen already in the 'De ære et æthere.'

\section{LAURA BENITEZ}

Instituto de Investigaciones Filosóficas

Universidad Nacional Autónoma de México

21 Ibid, 213. 